\title{
Design and Analysis of Low Frequency Communication System in Persian Gulf
}

\author{
H. Shahbazi, A. Karimifard \\ Institute of Engineering Research \\ Tehran, Iran
}

\begin{abstract}
One of the important applications of underwater communication is speech transmission between two divers or between divers and ship or submarine. This paper describes a project designed to investigate and demonstrate underwater communication system in Persian Gulf for speech transmission in a real channel. At first, transmitter is designed, then channel with real data is simulated by neural network and at last receiver is designed. Transmitted data is speech signal that for more safety in transmission and low frequency bandwidth, a cryptography algorithm and speech coding algorithm is applied in transmitter. Quadrature phase shift keying (QPSK) signaling is employed to make efficient use of the available channel bandwidth. In the receiver, linear equalizer and decision feedback equalizer (DFE) are tested and the best of them is applied. Also ray tracing method is used for simulation of sound waves propagation in Persian Gulf underwater communication channel.
\end{abstract}

\section{Introduction}

The underwater acoustic communication channels, however, have limited bandwidth, and often cause signal dispersion in time and frequency [1]. In this paper we have designed transmitter and receiver that transmit speech signal in the safe mode and low bandwidth with low bit error rate (BER) in Persian Gulf underwater communication channel.

In the first section, transmitter is designed. Because of strong frequency attenuation, channel bandwidth is limited. Therefore in transmitter, we have used LPC-10 (Linear Prediction Coder-10) algorithm to compressing speech signal. After compressing, for safety in transmission, RC5 (Rivest Cipher) cryptography algorithm has used to encrypt data. Then, for reduction of BER, channel coding is used. In the last section of transmission, data is modulated.

In third section, Persian Gulf underwater channel is simulated. In channel modeling, speed and depth profiles of Persian Gulf have used that have measured in 358 zone in Persian Gulf by Canadian in 2001.

In fourth section, linear equalizer and DFE equalizer are designed.

In fifth section, transmitter, Persian Gulf communication channel and receiver are simulated for real condition and in the last section, the results are presented.

\section{Transmitter}

In this section, transmitter has designed. Fig. 1, shows transmitter block diagram [2]. In the continuation, we will describe any block.

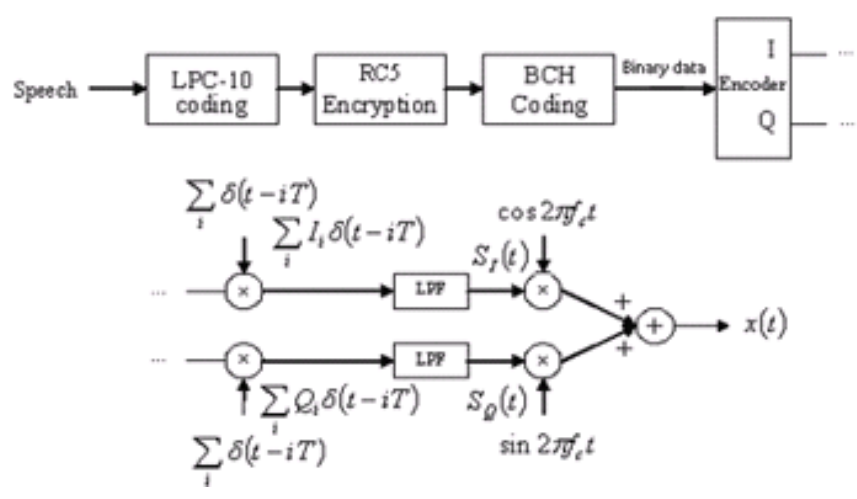

Figure 1. Transmitter block diagram

\section{A. Speech signal processing}

In the first block of transmitter, speech signal is compressed with LPC-10 algorithm. Sampling frequency is $8 \mathrm{KHz}$ and for any sample 8 bit is appropriated. Therefore sampling bit rate is 64 kbps. LPC-10 algorithm reduce bit rate to $2.4 \mathrm{kbps}$, consequently small bandwidth is needed and frequency attenuation is reduced $[3,4]$.

\section{B. Cryptography}

In the second block, data is encrypted with RC5 algorithm. RC5 is a symmetric block cipher designed to be suitable for both software and hardware implementation. It is a parameterized algorithm, with a variable block size, a variable number of rounds and a variable-length key.

This provides the opportunity for great flexibility in both performance characteristics and the level of security. In this paper, block size has selected 32, number of rounds are 16 and key length is 10 [5].

\section{Channel coding}

This block is needed for error correction in channel. $\mathrm{BCH}$ (Bose, ray-Chaudhuri, Hocquenghem) coding has been selected for channel coding. The inputs of applied $\mathrm{BCH}$ code are 11 bits that convert to 31 bits and are transmitted in channel. This code can correct 5 or fewer random errors in receiver [6].

\section{Modulator}

Modulator utilize quadrature phase shift keyed (QPSK) modulation. The encoder accepts the sequence of the input binary data. It has two outputs; in-phase, I, and quadrature, Q. 
For each distinct pair of input binary data a unique combination of $I=\{ \pm 1\}$ and $Q=\{ \pm 1\}$ is assigned. We can use a complex notation $\mathrm{D}=\mathrm{I}+\mathrm{jQ}$ to describe these four points separated in the complex plane by $45^{\circ}, 135^{\circ}, 225^{\circ}$ or $315^{\circ}$. The encoded data stream of I and Q is then used to modulate a sequence of $\delta$ pulses Transmitted every signaling period T. To limit their bandwidth such modulated sequences are then low-pass filtered by LPFs. The same low pass filters are applied at the receiver. The in-phase and quadrature signals at the output of the transmitting low pass filter are:

$$
\begin{aligned}
& S_{I}(t)=\sum_{i} I_{i} h_{H}(t-i T) \\
& S_{Q}(t)=\sum_{i} Q_{i} h_{H}(t-i T)
\end{aligned}
$$

where $h_{H}(t)$ is the impulse response of the low pass filter. The filtered signals are then multiplied by a carrier frequency, added and transmitted through the Persian Gulf underwater acoustic channel.

In order to reduce inter-symbol interference (ISI) we use modified raised-cosine filters (LPFs) with a roll-off factor $\beta=1$ and impulse response $h_{H}(t)$. The transfer function of the baseband channel has the form:

$$
H(f)= \begin{cases}\sqrt{T} \cos \frac{\pi f T}{2} ; & -\frac{1}{T}<f<\frac{1}{T} \\ 0 & ; \quad \text { elsewhere }\end{cases}
$$

where $\mathrm{T}=1 / 1200 \mathrm{~s}$. The transfer function of both the transmitter filter and receiver filter is then $X^{l / 2}(f)$, and the impulse response of the assumed nonfading part of the baseband channel is thus:

$$
x(t)=\int_{-\infty}^{\infty} X(f) e^{j 2 \pi f t} d f
$$

Thus the impulse response of each filter is obtained through the inverse Fourier transform of Eq. (2), which is:

$h_{T}(t)=h_{R}(t)=\frac{1}{\sqrt{T}}\left\{\frac{\sin \pi\left(\frac{2 t}{T}+\frac{1}{2}\right)}{\pi\left(\frac{2 t}{T}+\frac{1}{2}\right)}+\frac{\sin \pi\left(\frac{2 t}{T}-\frac{1}{2}\right)}{\pi\left(\frac{2 t}{T}-\frac{1}{2}\right)}\right\}$

Fig. 2 shows the impulse response of the raised cosine filter.

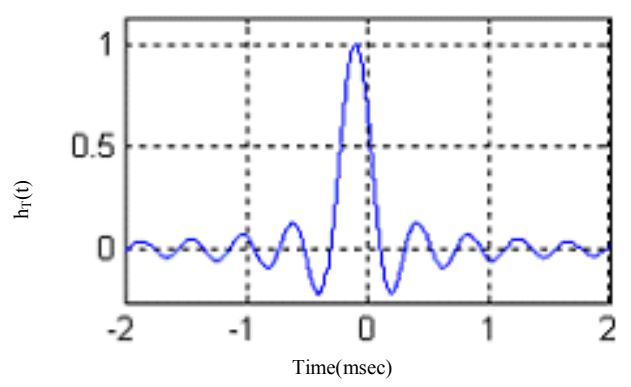

Figure 2. Impulse response of the raised cosine filter

To reduce sidelobe levels, the impulse response of the filter is modified by multiplying it with a Hamming window given as:

$$
w_{H}(t)=0.54+0.46 \cos \left(\frac{\pi t}{T}\right)
$$

Fig. 3 shows Hamming window in one period. The period of Hamming window is $2 \mathrm{~T}$ that $\mathrm{T}$ is the period of each symbol.

This window has $99.96 \%$ of its energy in the main lobe, with sidelobes of over $20 \mathrm{~dB}$ down from the peak, as shown in Fig. $4[7,8]$.

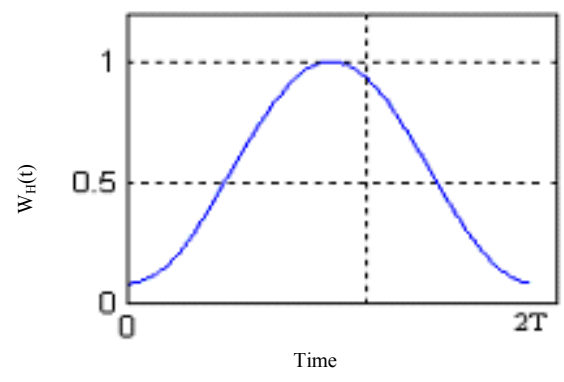

Figure 3. Hamming window in one period

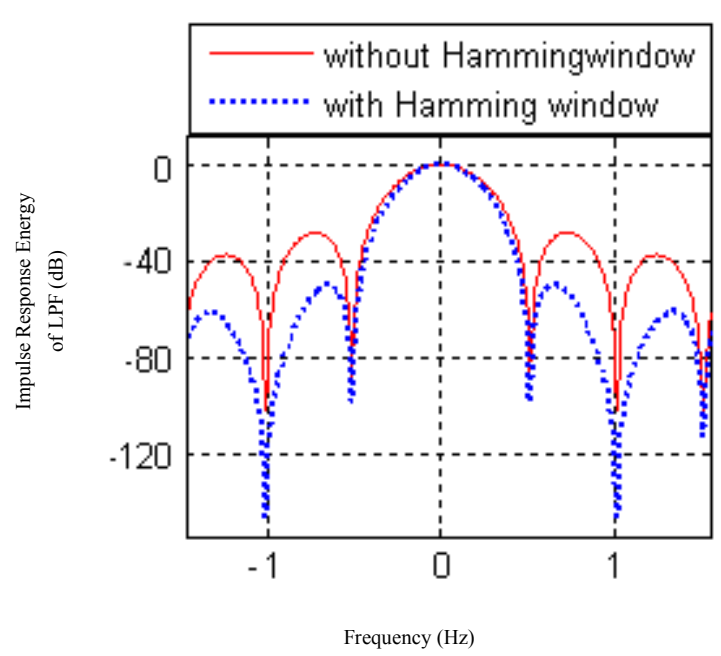

Figure 4. Impulse response of LPF with and without Hamming window 
Thus Impulse response of filters is corrected:

$$
h_{H}(t)=h_{T}(t) \cdot w_{H}(t)=h_{R}(t) \cdot w_{H}(t)
$$

We use this modified raised cosine filter in the transmitter and receiver. LPF outputs in transmitter are:

$$
\begin{aligned}
& S_{I}(t)=\sum_{i} I_{i} h_{H}(t-i T) \\
& S_{Q}(t)=\sum_{i} Q_{i} h_{H}(t-i T)
\end{aligned}
$$

For example if the input of LPF may be sequence 1011100011 then output has been shown in Fig. 5. The output signal of modulator has been shown in Fig. 6.

\section{Channel simulation}

There are many methods for acoustic wave propagation in underwater channel [9]. In this paper we have used ray method for acoustic wave propagation in Persian Gulf underwater channel $[10,11]$.

The salinity, depth and sound speed have been measured by Canadian in 358 points with different longitudes and latitudes in 2001. This information for other points has been estimated by neural network. At the first, we have designed a neural network that its inputs are longitudes and latitudes and its output is depth. Therefore with this neural network we can estimate depth in every point.

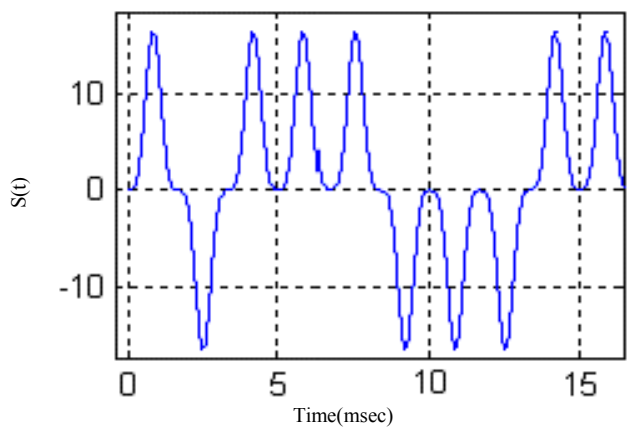

Figure 5. Output of LPF for sequence 1011100011

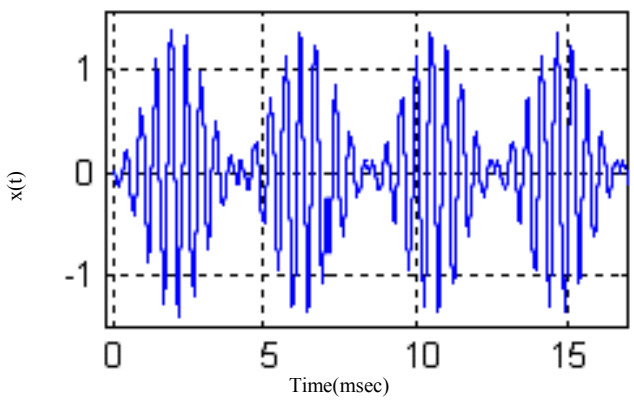

Figure 6. Output signal of modulator
Structure of this neural network has shown in Fig. 7. This network has two hidden layer with 10 neurons in each layer.

Too, other neural network has been designed for sound celerity estimation. The inputs of this neural network are longitude, latitude and depth and its output is sound celerity. With this neural network we can estimate sound celerity in every point. Neural network structure is shown in Fig. 8.

\section{Receiver design}

The digital acoustic communication is limited by severe inter-symbol interference (ISI) associated with long range, shallow underwater channels. The ISI is caused by multi-path propagation resulting from surface and bottom reflections. To overcome the effects of intersymbol interference, an adaptive equalization method employing a Mean Square Error (MSE) criterion is introduced. Computer simulations are carried out to verify the effectiveness of the equalization scheme for high data rate communication.

In the selection of an equalizer we consider convergence rate, processing complexity and the ability to track changes in the channel characteristics.

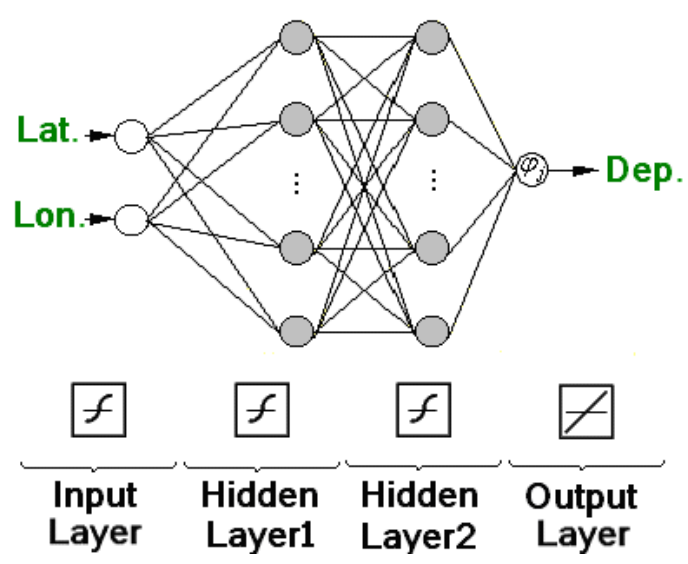

Figure 7. Designed neural network for depth estimation

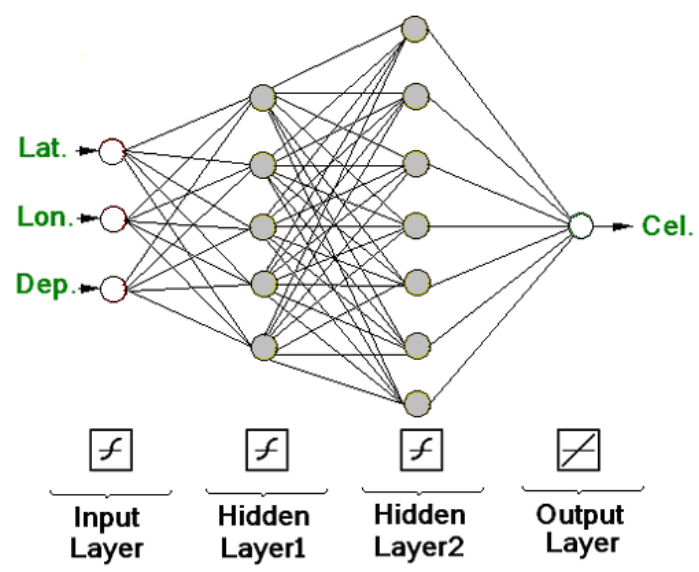

Figure 8. Designed neural network for sound celerity estimation 
There are a variety of algorithms which have been investigated for use in underwater acoustic communication.

In this paper we have tested linear and DFE equalizers for speech signal transmitting in Persian Gulf underwater channel.

\section{A. Linear equalizer}

A symbol-spaced linear equalizer consists of a tapped delay line that stores samples from the input signal. Once per symbol period, the equalizer outputs a weighted sum of the values in the delay line and updates the weights to prepare for the next symbol period. This class of equalizer is called "symbol-spaced" because the sample rates of the input and output are equal.

Fig. 9 is a schematic of a symbol-spaced linear equalizer with $\mathrm{L}$ weights, where the symbol period is $\mathrm{T}$.

Impulse response of linear equalizer is:

$$
H_{e q}(z)=w_{1}+w_{2} z^{-1}+\ldots+w_{L} z^{-L+1}
$$

We should determine $\left\{w_{i}\right\}_{i=1}^{L}$ to minimize error probability. The criterion used in the optimization of the equalizer coefficients is the minimization of the mean square error (MSE), that is [12]:

$$
\begin{aligned}
& \left.\min \left(\left.|E| e(k T)\right|^{2}\right\rfloor\right)= \\
& \min \left(\left[E\left|z_{d}(k T)-z(k T)\right|^{2}\right]\right)
\end{aligned}
$$

Where $z_{d}(k T)$ and $z(k T)$ are the actual transmitted data and the equalizer output at $\mathrm{t}=\mathrm{kT}$, respectively. For an ideal channel, $d(k T)=z(k T)$. During the equalizer training period, a block of data known to the receiver can be transmitted and used to adjust the equalizer coefficients. After the training period a receiver does not know the actual transmitted data so that the adjustment of the equalizer coefficients (tap gains) is performed using the decisions at the output of the detector (decision-directed). This is symbolized by switch $\mathrm{S}$ in Fig. 9. The minimization of the MSE is accomplished recursively by the use of the stochastic gradient algorithm, called the least mean square (LMS) algorithm.

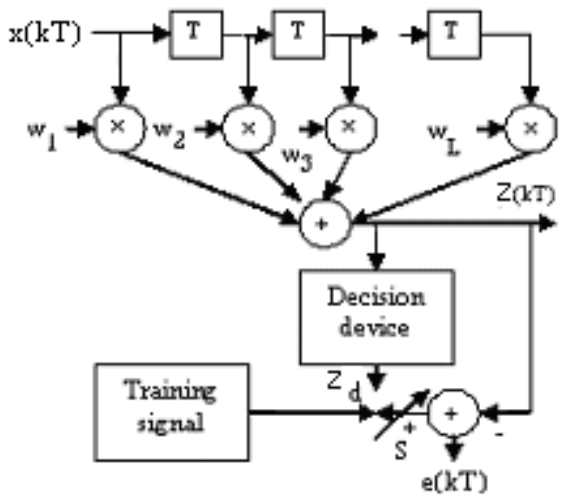

Figure 9. Symbol-spaced linear equalizer
New set of coefficients is obtained iteratively as:

$$
w_{\text {। }}[(k+1) T]=w_{\text {। }}(k T)+\Delta e(k T) y^{*}(k T-\mathrm{I} T)
$$

where $\Delta$ is a positive adaptation constant (step size), $e(k n)=z_{d}(k T)-z(k T)$ is the error signal and * denotes the complex conjugate.

The step-size parameter $\Delta$ controls the rate of adaptation of the equalizer and stability of the stochastic gradient algorithm. Larger $\Delta$ leads to more rapid convergence, but also leads to larger fluctuations in the equalizer coefficients during steady state operation. The selection of $\Delta$ is therefore a compromise between rapid convergence and small variance of self noise.

\section{B. Decision feedback equalizer}

A decision-feedback equalizer is a nonlinear equalizer that contains a forward filter and a feedback filter. The forward filter is fractionally spaced with spacing $\mathrm{T} / 2$; while the feedback filter contains a tapped delay line whose inputs are the decisions made on the equalized signal. The purpose of a DFE is to cancel intersymbol interference while minimizing noise enhancement. By contrast, noise enhancement is a typical problem with the linear equalizers described earlier. Fig. 10 shows structure of decision feedback equalizer (DFE).

Similar to linear equalizer, the criterion used in the optimization of the equalizer coefficients is the minimization of the mean square error (MSE) by use of the least mean square (LMS) algorithm. The output of DFE at $t=k T$ is given as $[13,14,15]$ :

$$
\begin{aligned}
z(k T)= & \sum_{n=0}^{N-1} c_{n}(k T) y(k T-n T / 2)- \\
& \sum_{m=1}^{M} b_{m}(k T) d(k T-m)
\end{aligned}
$$

And error is:

$$
e(k T)=d(k T)-z(k T)
$$

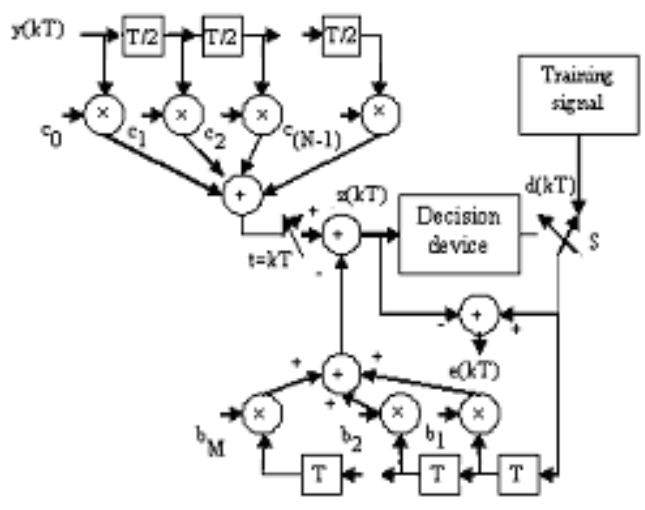

Figure 10. Structure of decision feedback equalizer 
New set of coefficients is obtained iteratively as:

$$
\begin{aligned}
& c_{n}[(k+1) T]= \\
& c_{n}(k T)+\Delta e(k T) y^{*}(k T-n T / 2) \\
& b_{m}[(k+1) T]= \\
& b_{m}(k T)+\Delta e(k T) d^{*}(k T-m T)
\end{aligned}
$$

\section{Simulation results}

In this section, we simulate Persian Gulf underwater channel in September 2001 and both linear and DFE equalizers are tested in receiver. Table I shows the parameters of the channel and system.

TABLE I

SIMULATION PARAMETERS

\begin{tabular}{|l|l|}
\hline Range & $10 \mathrm{~km}$ \\
\hline Water depth & $120 \mathrm{~m}$ \\
\hline Transmitter depth & $15 \mathrm{~m}$ \\
\hline Receiver depth & $30 \mathrm{~m}$ \\
\hline Carrier frequency & $10 \mathrm{kHz}$ \\
\hline Bandwidth & $2 \mathrm{kHz}$ \\
\hline
\end{tabular}

Fig. 11 shows the sound speed profile in Persian Gulf in September 2001 and Fig. 12 shows directions of sound wave propagation in Persian Gulf underwater channel. Fig. 13 shows the length of any wave propagation path vs. meter and Fig. 14 shows the attenuation of any path. The longer paths have more attenuation. The path that is longer, its attenuation is more. By dividing rang to sound speed in water, we can calculate the delay of each path.

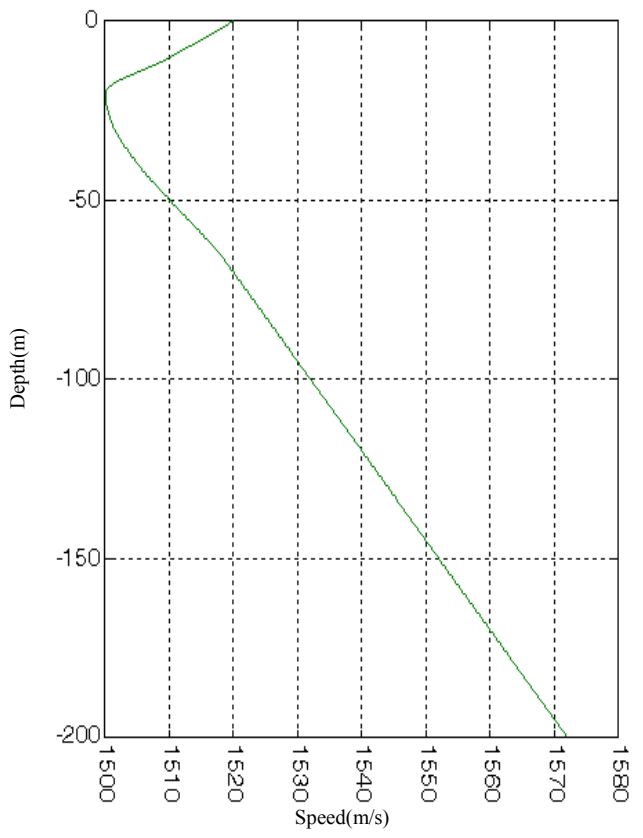

Figure 11. Speed profile in September 2001 in Persian Gulf at longitude $59^{0}$ and latitude $24^{\circ}$

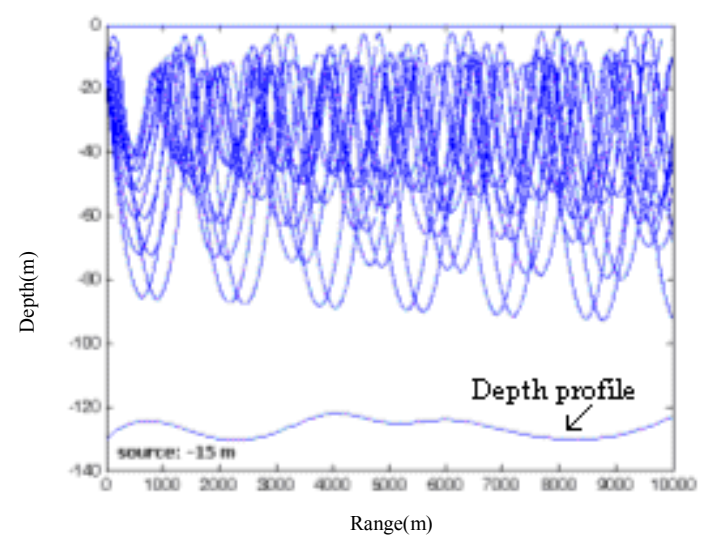

Figure 12. Direction of sound wave propagation in Persian Gulf underwater channel

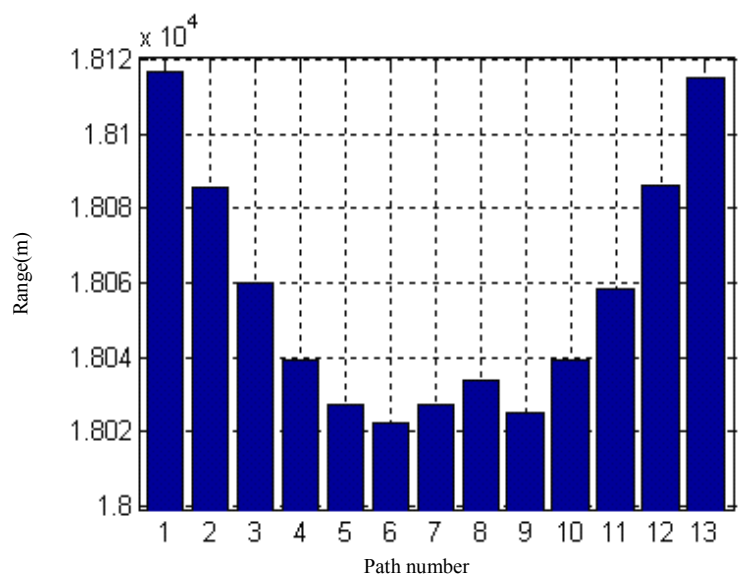

Figure 13. Range of paths

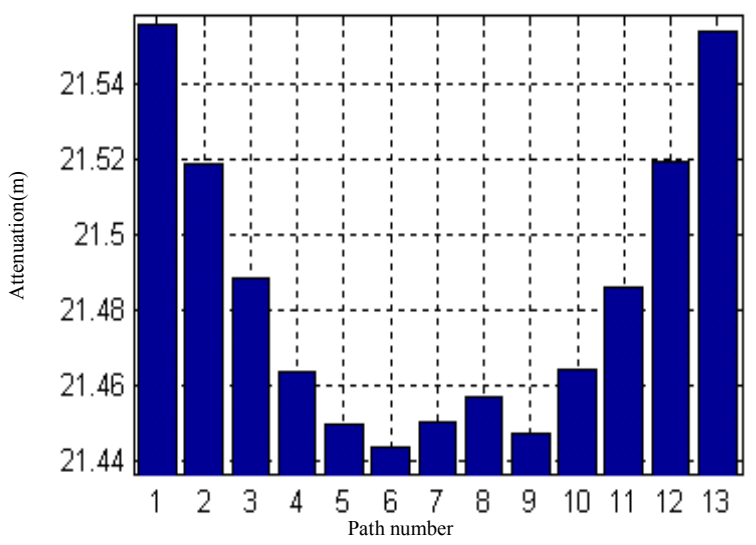

Figure 14. Attenuation of paths

Structure of the receiver has been shown in Fig. 15. We can replace adaptive equalizer block with linear or nonlinear (DFE) equalizer. The output of channel $r(t)$ and outputs of LPF in receiver are shown in Fig. 16, Fig. 17 and Fig. 18. 

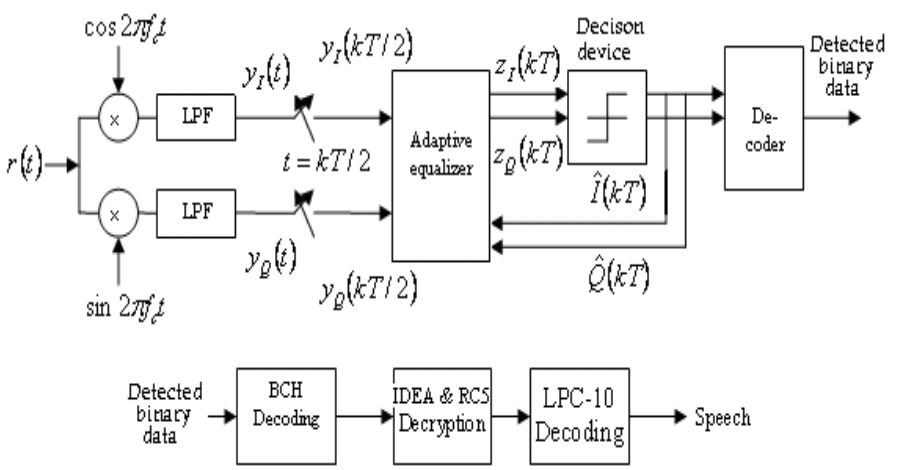

Figure 15. Structure of the receiver

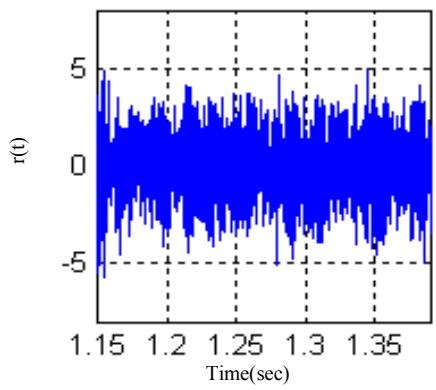

Figure 16. Output of channel

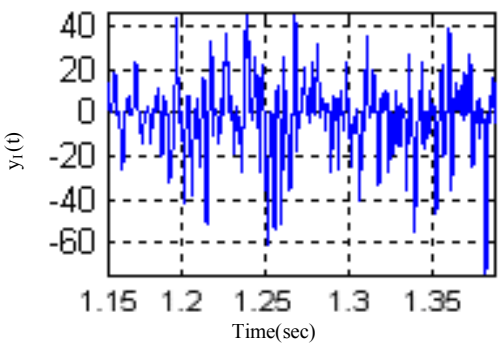

Figure 17. Output of LPF in receiver

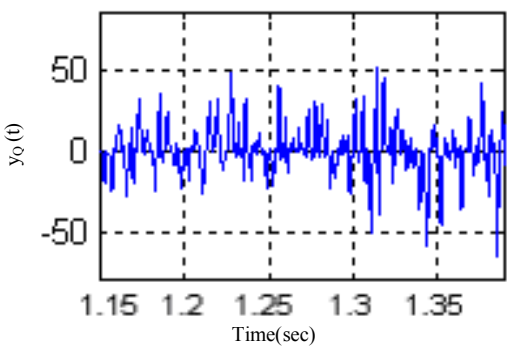

Figure 18. Output of LPF in receiver

With respect to maximum delay in channel, linear equalizer has 46 weights and $\Delta=10^{-4}$. DFE has 92 weights in forward filter and 46 weights in backward filter.

2500 symbol is applied for equalizers training. As can be seen in Fig. 19, for convergence, the equalizers requires over 100 iterations.
The output of linear and DFE equalizer in $\mathrm{SNR}=0 \mathrm{~dB}$ have been shown in Fig. 20 and Fig. 21.

In Fig. 22 and Fig. 23, we have shown speech signal in transmitter and receiver.

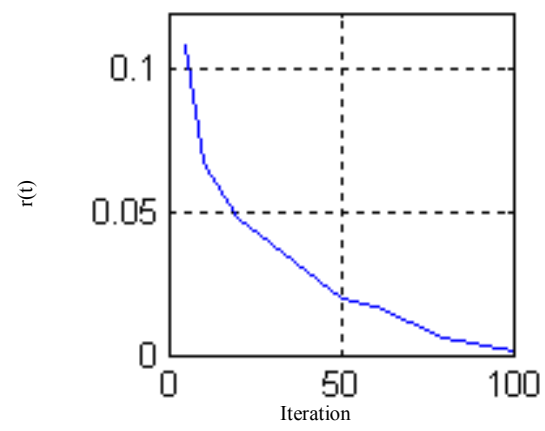

Figure 19. BER vs. number of iterations

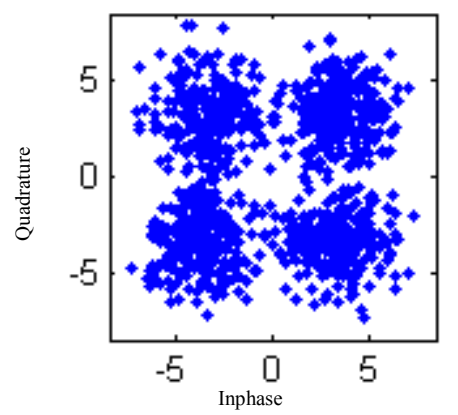

Figure 20. Output of linear equalizer in $\mathrm{SNR}=0 \mathrm{~dB}$

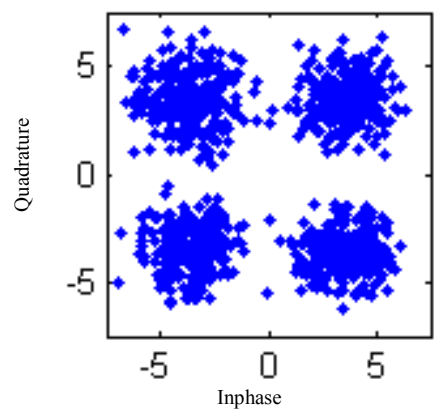

Figure 21. Output of DFE in $\mathrm{SNR}=0 \mathrm{~dB}$

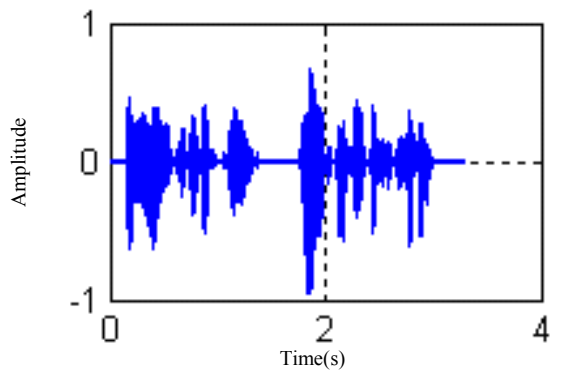

Figure 22. Speech signal in the transmitter 


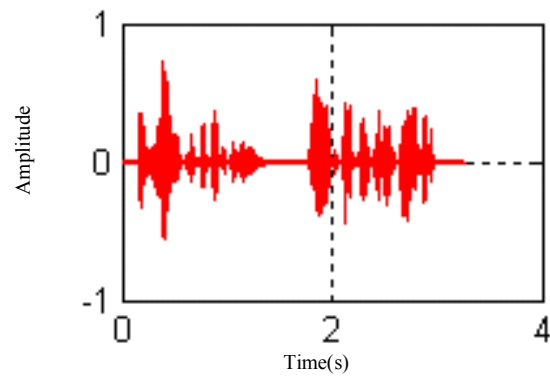

Figure 23. Speech signal in the receiver

\section{Results}

In this paper, we designed an underwater communication system in Persian Gulf. In transmitter, data are compressed, encrypted and modulated for sending in underwater channel. Then we simulated wave propagation in Persian Gulf underwater channel by using neural network and ray method. In receiver, we tested linear and decision feedback equalizer. Results show that DFE is suitable for Persian Gulf underwater channel. Also data are decrypted and decoded.

\section{REFRENCES}

[1] M. Stojanovic, "Underwater Acoustic Communications, " invited paper, Proc. IEEE Electro International Conference, Boston, MA, pp. 435-440, June 1995.

[2] F. Xiong, Digital Modulation Techniques, Artech House, Boston, USA, 2000.

[3] A. S. Spanias, “Speech Coding," Proc. IEEE, Vol. 82, No. 10, pp. 15411582 Oct. 1994.

[4] T. Painter and A. S. Spanias, "Perceptual Coding of Digital Audio,"Proc. IEEE, Vol. 88, No. 4. pp. 451-515, Apr. 2000.

[5] M. Y. Rhee Internet Security, John Wiley \& Sons Ltd, 2003.

[6] B. Stephen, Error Control Systems for Digital Communication and Storage, Upper Saddle River, N.J., Prentice Hall, 1995.

[7] M. C. Jeruchim, P. Balaban and K. S. Shanmugan, Simulation of Communication Systems, Plenum Press, New York, 1992.

[8] A. Falahati, B. Woodward and S. C. Bateman, "Underwater Acoustic Channel Models For 4800 b/s QPSK Signals," IEEE Journal of Oceanic Engineering, Vol. 16, No. 1, pp. 12-20, Jan. 1991.

[9] R. J. Urick. Principles of Underwater Sound. McGraw-Hill Book Company, third edition, 1983.

[10] K. C. Hegewisch, N. R. Cerruti and S. Tomsovic, "Ocean acoustic wave propagation and ray method correspondence: internal wave fine structure," The Journal of the Acoustical Society of America, Vol. 117, No.3, pp. 1582-1594, Oct. 2005.

[11] M. K. Simon, M. S. Alouini, Digital Communication Over Fading Channels, John Wiley \& Sons Ltd, 2000.

[12] J. G. proakis, Digital Communications, 3rd ed. Boston, MA McGraw Hill, 1995.

[13] J. G. Proakis, M. Stojanovic and J. Catipovic, "Phase coherent digital communications for underwater acoustic communications," IEEE Journal of Oceanic Engineering. Vol. 19, pp. 100-111, Jan. 1994.

[14] M. Stojanovic, "Recent Advances in High-Speed Underwater Acoustic Communications, " IEEE Journal of Oceanic Engineering, Vol.121, No.2, pp.125-136, April 1996.

[15] J. P. Gomes and V. Barroso, "SDR Underwater Acoustic Modem," Ph.D Thesis, Instituto de Sistemas e Robotica, Lisboa, Portugal, Apr. 2005. 